\title{
The Modified National Early Warning Score (m-NEWS) for COVID-19-Infected Patient Evaluation: a Proof-of-Concept
}

\author{
Luca Galassi ${ }^{1}$ (1) - Daniele Schena ${ }^{1}$ \\ Accepted: 17 December 2020 / Published online: 3 January 2021 \\ (C) The Author(s), under exclusive licence to Springer Nature Switzerland AG part of Springer Nature 2021
}

\begin{abstract}
The second wave of Sars CoV 2 pandemic in Italy still represents an important concern for the Italian health system (IHS). Despite the experience gained during the first phase of the pandemic in January 2020, COVID-19 patients are still arising, putting on a strain resources of local authorities and emergency services. The aim of this research project is to analyze the feasibility of using the modified National Early Warning Score (m-NEWS) as a way to rapidly identify COVID-19-infected patient with high risk of clinical deterioration, helping the clinical pre-evaluation in a moment of overwhelming request for the Italian out-ofhospital emergency system (Perlini et al., Intern Emerg Med 15(5):825-833, 2020) as during the initial coronavirus outbreak.
\end{abstract}

Keywords SARS CoV2 $\cdot$ Novel coronavirus $\cdot$ Modified National Early Warning Score · COVID-19

Although the first coronavirus cases in Italy can be dated back to mid-January 2020 [1], the novel Sars CoV2 outbreak in Italy has recently reached a new record of cases with 40,902 newly infected people on the 13 of November [2] evenly distributed all over the Italian territory.

The first wave that hit the northern regions of Italy and in particular Bergamo Hinterland caused important problems for the management of the critical number of COVID-19-infected patients with the collapse of the most part of hospitals emergency departments, ordinary internal medicine wards, as well as critical care units [3].

Currently, we are witnessing the second wave of the pandemic while still trying to find solutions to manage a progressive increase in the number of COVID-19 cases [4]. To date, Italy has reached a total of 1,205,881 confirmed cases and has reported 45,733 deaths due to COVID-19 infection [5].

The lack of medical specialists trained for the critical care management of patients combined with hospital insufficiencies and shortcomings in medical facilities is slowly aggravating the clinical situation. In addition, the introduction of local regional lockdowns or closure of high schools due to an

This article is part of the Topical Collection on COVID-19

Luca Galassi

lucagalassimd@gmail.com

1 Orthopedics and Traumatology Unit, A.O. ASST Bergamo Est, P.O. Pesenti Fenaroli, Alzano Lombardo, Bergamo, Italy outbreak of contagions does not appear to have helped stem the tide of new cases [6].

Despite all of the measures taken in order to slow down the spread of COVID-19, the number of infected patients continues to rise and with it and increased pressure on emergency services and departments all over Italy.

In order to guarantee the best level of care, our main objective is to develop a fast and simple scorecard that can be easily used by general practitioners across the country on COVID-19-infected patients.

Correspondingly with this innovative approach, we have started a research project in order to evaluate the potential usage of modified National Early Warning Score (m-NEWS), already hypothesized during the first COVID-19 epidemic in Wuhan [7] and suggested in other review [8], as a tool to be used in the pre-evaluation of COVID-19 patients even before their possible hospitalization.

The use in primary care of m-NEWS score could quickly identify patients at high risk of clinical deterioration, indicating whenever a patient may need early hospitalization, critical care, or can be treated at home by their general practitioner.

m-NEWS score may be adopted as a common and standardized language to rapidly describe patients' clinical conditions among different healthcare professionals (GP, nurses, ambulance service, or the emergency department admitting team).

This rapid and simple score [9] can perfectly fit an emergency situation in which GP may have only one occasion to visit patients (due to the large volume of cases and to limit the 
number of Sars-CoV2-related contagions) and decide their diagnostic and therapeutic process.

The calculation of the score, together with the full clinical context of the patient, will prioritize and triage the patients that need fast admission and transfer to hospital and critical care helping in managing a large volume of patients as it happened in the COVID-19 epidemic.

Although m-NEWS score can give important information for clinical assessment of COVID-19-infected patients, the score should be always interpreted within the full clinical context of the patient and could not replace clinician's ability to properly assess the acuity of the patient $[10,11]$.

We are inviting general practitioners and COVID-19dedicated hospitals to use the m-NEWS score during this second wave of the epidemic in the attempt to obtain multicentric data that could provide further evidence to encourage the innovative use of this tool, which in turn would eventually lighten the workload on hospitals and hopefully reduce the number of deaths.

Authors' Contribution All the authors had given important scientific contribution to this study considering all aspect of it: ideation, writing, and final check, and we have read the entire manuscript and take responsibility for its content.

\section{Compliance with Ethical Standards}

Conflict of Interest The authors declare that they have no conflict of interest.

\section{References}

1. Zehender G, Lai A, Bergna A, Meroni L, Riva A, Balotta C, et al. Genomic characterization and phylogenetic analysis of SARSCOV-2 in Italy. J Med Virol. 2020;92:1637-40. https://doi.org/ 10.1002/jmv.25794.

2. Italian Ministry of Interior. Covid-19 - Situazione in Italia. http://www. salute.gov.it/portale/nuovocoronavirus/ dettaglioContenutiNuovoCoronavirus.jsp?lingua=italiano\&id= $5351 \&$ area $=$ nuovoCoronavirus \&menu=vuoto. Accessed 18 Nov 2020.
3. Armocida B, Formenti B, Ussai S, Palestra F, Missoni E. The Italian health system and the COVID-19 challenge. Lancet Public Health. 2020 May;5(5):e253. https://doi.org/10.1016/S24682667(20)30074-8.

4. Paterlini M. Covid:19: Italy has wasted the sacrifices of the first wave, say experts. BMJ. 2020;371:m4279. https://doi.org/10.1136/ bmj.m4279.

5. World Heath Organisation. Italy COVID19 confirmed cases. https://covid19.who.int/region/euro/country/it. Accessed 18 Nov 2020.

6. Italian Ministry of Interior. Ulteriori misure urgenti in materia di contenimento e gestione dell'emergenza epidemiologica da COVID19. https://www.gazzettaufficiale.it/eli/gu/2020/11/14/284/ sg/pdf. Accessed 13 Nov 2020.

7. Liao X, Wang B, Kang Y. Novel coronavirus infection during the 2019-2020 epidemic: preparing intensive care units - the experience in Sichuan Province, China. Intensive Care Med. 2020;46: 357-60. https://doi.org/10.1007/s00134-020-05954-2.

8. Pennica A, Conforti G, Falangone F, Martocchia A, Tafaro L, Sentimentale A, et al. Clinical management of adult coronavirus infection disease 2019 (COVID-19) positive in the setting of low and medium intensity of care: a short practical review. SN Compr Clin Med. 2020;2:1-6. https://doi.org/10.1007/s42399-020-00333-w.

9. Scott LJ, Redmond NM, Tavaré A, Little H, Srivastava S, Pullyblank A. Association between national early warning scores in primary care and clinical outcomes: an observational study in UK primary and secondary care. Br J Gen Pract. 2020;70(695): e374-80. https://doi.org/10.3399/bjgp20X709337.

10. Brangan E, Banks J, Brant H, Pullyblank A, Le Roux H, Redwood S. Using the national early warning score (NEWS) outside acute hospital settings: a qualitative study of staff experiences in the West of England. BMJ Open. 2018;8(10):e022528. Published 2018 Oct 27. https://doi.org/10.1136/bmjopen-2018-022528.

11. Perlini S, Canevari F, Cortesi S, et al. Emergency department and out-of-hospital emergency system (112-AREU 118) integrated response to coronavirus disease 2019 in a northern Italy centre. Intern Emerg Med. 2020;15(5):825-33. https://doi.org/10.1007/s11739020-02390-4.

This manuscript is not currently under consideration, in press, or published elsewhere. This manuscript is truthful original work without fabrication, fraud, or plagiarism.

Publisher's Note Springer Nature remains neutral with regard to jurisdictional claims in published maps and institutional affiliations. 